\title{
Adapting Collaborative Approaches for Service Provision to Low-Income Countries: Expert Panel Results
}

\author{
Kimberly Pugel ${ }^{1,2, *} \mathbb{D}$, Amy Javernick-Will ${ }^{1,2} \mathbb{D}$, Matthew Koschmann ${ }^{3}$, Shawn Peabody ${ }^{2,4}$ and \\ Karl Linden 1,2 \\ 1 Department of Civil, Environmental, and Architectural Engineering, University of Colorado Boulder, \\ Boulder, CO 80309, USA; amy.javernick@colorado.edu (A.J.); karl.linden@colorado.edu (K.L.) \\ 2 USAID Sustainable WASH Systems Learning Partnership, United States Agency for International \\ Development, Washington, DC 200004, USA; speabody@enviroincentives.com \\ 3 Department of Communication, University of Colorado Boulder, Boulder, CO 80309, USA; \\ matthew.koschmann@colorado.edu \\ 4 Environmental Incentives, Washington, DC 20005, USA \\ * Correspondence: kimberly.pugel@colorado.edu
}

Received: 10 March 2020; Accepted: 23 March 2020; Published: 25 March 2020

\begin{abstract}
The international development sector is increasingly implementing collaborative approaches that facilitate a range of sectoral-level stakeholders to jointly address complex problems facing sustainable public service delivery, for which guidance does not explicitly exist. The literature on collaborative approaches has been built on experiences in high-income countries with vastly different governance capabilities, limiting their global relevance. A Delphi expert panel addressed this need by evaluating 58 factors hypothesized in the literature to contribute to the success of collaborative approaches. The panel rated factors according to their importance in low-income country contexts, on a scale from Not Important to Essential. Experts agreed on the importance of 49 factors, eight of which were essential for success. Rich qualitative data from open-ended responses revealed factors that may be unique to low-income country contexts and to service delivery applications, including how government capacity, politics, donor influence, and culture can influence decisions on structuring leadership and facilitation roles, appropriately engaging the government, and building legitimacy. Key considerations for future practice and research are summarized in a table in the appendix. This study contributes to both literature and practice by identifying the relative importance of factors to consider when designing collaborative approaches in low-income countries with limited governance capabilities.
\end{abstract}

Keywords: collaborative approaches; service provision; low-income countries; collective action; collective impact

\section{Introduction}

Around the world, local governments are mandated to provide certain public services, such as water, sanitation, health, energy, transportation or education, but in many countries, they do so poorly. This could be due to budget or capacity challenges or result from poorly-defined roles, responsibilities, or governance structures for service provision. In response to this limited effectiveness, non-state actors (e.g., private sector, civil society organizations, international NGOs) often intervene to supplement services [1-3], yet do not typically coordinate their efforts. 'Collaborative approaches' seek to strengthen the collaboration among sectoral-level stakeholders, government, and other relevant entities in order to collectively address the barriers limiting service quality, see Reference [4]. As a result, development 
practitioners are increasingly interested in implementing collaborative approaches to strengthen the accountability of local governments to provide services [5,6]. However, existing theory and literature has been built on experiences in high-income countries whose governments can more effectively implement sound policies and mandates to provide and regulate public services. Development practitioners, thus, lack applicable guidance on how to facilitate these approaches in contexts with more 'limited' governance. By 'limited governance', we are referring to contexts where the government is less effective at providing services, where regulatory capacity is constrained, where political instability and corruption are more prevalent, and where citizen voice and accountability is weaker $[7,8]$. It is these contexts with limited governance where a large swath of informal and non-governmental service providers step in, where multi-lateral donors heavily influence local government agendas, and in many cases where there is a need for greater coordination and collaboration to sufficiently provide a public service. According to the World Bank Worldwide Governance Indicators, low-income country governments tend to be less effective at providing quality public services than upper-middle income and high income countries [7,9], and thus, throughout this paper, we will refer to these limited-governance contexts as low-income country contexts. However, we recognize that other datasets, such as the Ibrahim Index of African Governance, show that governance capabilities range widely amongst low-income countries [8]. Development practitioners nonetheless need to find ways to adapt collaborative approaches to work within these limited-governance contexts in low-income countries. For example, implementers have needed to adapt the traditional collective impact framework [10] to work more appropriately in their contexts of Ghana, Uganda, and Cambodia for water and sanitation service provision [11], but there is little documentation of what those changes were and why they were made. To formally document some of these adaptations and provide better guidance to development practitioners, we convened a panel of experts to evaluate the importance of a series of factors that influence collaborative approaches for service delivery in limited-governance contexts.

\subsection{Literature Gaps}

This study seeks to fill two gaps in the literature that currently limit development practitioners from directly applying lessons and guidance from the plethora of literature bodies on collaborative approaches. First and foremost, existing literature lacks evidence from limited-governance contexts, such as low-income countries, and from service delivery applications. This gap leads to questions about which factors are universal and may influence collaborative approaches in any context versus which factors are specific to certain contexts. Second, the existing bodies of literature on collaborative approaches remain largely disconnected and lack standard terminology for core tenants of their work. This gap creates challenges for implementers seeking to find guidance on how to set up their collaborative approach the "right" way, for funders or researchers seeking to reference similar work, and for the collaborative itself to build legitimacy with its members, service users, and governments.

\subsubsection{Literature Lacks Evidence of Collaborative Approaches in Low-Income Countries}

The scope, uncertainty, and complexity of many problems throughout the world often necessitate collective work between organizations and across sectors, yet many of these entities bring differing priorities, funding constraints, and ways of working to the table. Collective work comes in many forms, ranging from 'cooperation' to 'coordination' to 'collaboration' and beyond. Colloquially, these terms are often used interchangeably, but in recent years, literature has delineated between them. Cooperation most commonly refers to parties helping each other out, rather than competing. When those parties begin to align specific tasks toward a shared interest, such as signing a Memorandum of Understanding, they are coordinating with one another [12-14]. Collaborative work, then, is when these entities move further to enter into a new, interdependent way of working: Giving up some of their autonomy, taking on shared risks, and becoming accountable to one another. Collaborative work can be undertaken to "tackle knotty societal problems, to promote innovation, to provide public services, to expand governance capabilities, to set standards for a field, or to resolve conflicts that 
impede progress on critical issues" [15] (pp. 1-2). For this work we define a collaborative approach for service delivery as a structure and a process where sectoral stakeholders who play a role in service provision regularly convene and take joint actions to address shared problems, in which: (a) Problems are complex, and their solutions require deliberation and collective action by many actors; and (b) stakeholders clarify responsibilities and hold each other accountable for actions. This is similar to, and builds off of, many definitions of collaborative work used across literature [13,15-21], but is specific to service delivery.

To date, most studies of collaborative approaches for service provision are focused on high-income contexts within the United States, Australia, Canada, and European countries [12]. Limited-governance contexts can certainly exist within high-income countries; however, the premise of this work lies in the assumption that more broadly, these contexts have stronger governance regimes for service provision and regulation [22,23], the state effectively carries out their mandate to provide services [24], citizens are relatively individualistic and do not accept large power hierarchies [25,26], programs are typically funded by domestic funding sources, and contexts are not influenced with international donors and non-governmental actors. These differences matter for structuring collaborative approaches, and thus, have spurred development practitioners to adapt existing knowledge to better fit their work; each is described in more detail below.

Service provision environments in limited-governance contexts, particularly in Africa, have a very different history and face very different challenges than those in North America, Australia, and Europe [27-29]. For example, in low-income countries, roughly forty percent of the population do not have basic water services, while roughly seventy percent do not have access to basic sanitation services [30], and those with services often lose those services when their water or sanitation infrastructure fails, due to insufficient maintenance [31]. Many believe that strengthening local governments to provide these as public services is the best route to reach universal access. Scholars and development agencies, such as the U.S. Agency for International Development (USAID) and the Sustainable Development Goals Center for Africa, for example, are promoting agendas seeking to strengthen local and national governments to be more capable and accountable for providing services, becoming more 'self-reliant' [5,6,22,32], and allowing development to be government-led [8]. This means that it is important for collaborative approaches in limited-governance contexts to be designed to put local governments at the forefront of service provision and governance [33]. However, this means that implementers will face the inefficiencies and challenges inherent to the public sector in Africa, such as "poor staff morale, decline in work effort, absenteeism, moonlighting and 'daylighting'; difficulty in recruiting and retaining technical and professional staff ... The entrenched debility of the sector, it is alleged, makes it extremely difficult for even well intentioned public officials to be effective" as Owusu and Ohemeng [22] point out (p. 121). Another important consideration related to how to involve government is what role they should play in service delivery. In development, though the private sector continues to play an important role as a service provider, the role of service authority is increasingly emphasized as a government role, especially in the water and sanitation sector $[34,35]$. As a result, adequate government support and leadership in collaborative approaches may be critical for being seen as legitimate and viable, as well as for the results to scale. In a recent blog post, the collective impact literature began discussing the importance of government involvement when applying collective impact approaches to development contexts [36]; however, this is the only case we could find where this is discussed. 
Running contrary to these current development paradigms, many collaborative approaches in high-income countries have been designed to shift service provider and service authority responsibilities onto the shoulders of the private sector, organizations, or communities, reflecting a "trend among government agencies toward outsourcing the provision of services" [37] (p. 422). This may be "due to a broad disillusionment in the ability of governments around the world to solve society's problems, causing people to look more closely at alternative models of change" [38] (p. 3). This 'outsourcing' is often achieved through collaborative approaches, as described in Talesh's Handbook on Theories of Governance [39]: "Non-governmental actors are taking a more active role in governing themselves and trying to maintain a public good ... public agencies are directly engaging non-state actors in collective decision-making. These co-regulatory schemes are consensus-oriented and deliberative and aim to allow private industry more direct involvement and control in implementing public policies" (p. 105). This means that the wide berth of literature on collaborative approaches may be designed for shifting service authority roles away from government, and thus, likely underemphasizes key strategies for government engagement.

Finally, cultural attitudes, such as those investigated by Hofstede [25] (e.g., individualist versus collectivist societies), also may affect the formation of collective arrangements. For example, a collaborative approach that requires equal, non-hierarchical distributions of power may be less accepted in countries with high power distance scores, such as Ethiopia and Kenya. Similarly, strategies employed in individualistic cultures to incentivize organizations and agencies to pursue collective goals may be less necessary for collectivist cultures.

\subsubsection{Literature Remains Largely Disconnected and Lacks Standard Terminology}

Collaborative work is an area of study covered by many bodies of literature, with each field contributing knowledge about how this style of work unfolds. These bodies of literature serve as a firm basis on which this study and future studies of collaborative approaches in low-income country contexts can build. The most applicable and relevant bodies of literature include inter-organizational collaboration [13,15,40-42], collaborative governance [16,18,19,43], institutional collective action [44], collaborative management [45], coalition building [37], community organizing [46-48], multi-stakeholder collaboration or partnerships [16,49-52], 'samhandling' [53], and collective impact $[10,38,54-58]$.

While this literature is extensive, it is largely disconnected. As such, each describes different strategies for setting up, running, and measuring the success of collaborative approaches. In some cases, some scholars from one body of literature declare themselves distinctly different from others. For example, the founders of the collective impact framework claim that collective impact "is not just a fancy name for collaboration, but represents a fundamentally different, more disciplined, and higher performing approach to achieving large-scale social impact" [38] (p. 2). These scholars differentiate collective impact initiatives from others by stating that they "involve a centralized infrastructure, a dedicated staff, and a structured process that leads to a common agenda, shared measurement, continuous communication, and mutually reinforcing activities among all participants" [10] (p. 38). However, almost every other type of collaborative approach cites the importance of having facilitated leadership, agreement on a vision and agenda, relationships and interaction between members, and for strategic, concerted actions to be taken together $[18,19,59]$. Ultimately, having a diversity of theoretical perspectives allows for more theory to be built, but in practice, it creates confusion and frustration for organizations or agencies seeking advice to set up their approach "the right way", for funding agencies who may desire to fund these approaches but cannot find adequate evidence or metrics to use to monitor success, and for researchers seeking to compare existing approaches to the wider body of literature. Inconsistencies in terminology also challenge the ability for implementers to build legitimacy with local stakeholders, service users, and governments. As the body of literature continues to advance and expand, advancements will struggle to be applied and adopted in practice without 
commonly accepted definitions of terminology, implementation decisions, or methods for tracking progress and outcomes.

A leading researcher in the field of inter-organization collaboration, Chris Huxham [20], pointed to this disconnect in the literature almost two decades ago, stating that "there appears to be little mutual recognition of research across disciplines and paradigms, so there tends to be little overlap in the articles that are cited in reference lists. The complexity of perspectives can be baffling, making it difficult for individual researchers to interpret material fully that is coming from another perspective" (p. 402). Unfortunately, this is still the case today, which limits the ability of practitioners to fully interpret lessons and guidance that these bodies of literature aim to provide.

\subsection{Point of Departure and Research Question}

The root of the problem lies within a web of systemic challenges: Local governments in low-income countries are often mandated to provide services without adequate regulatory structures or resources; national and global development agendas, mandates, and funding mechanisms are diverse and often conflicting; non-governmental service providers step in to informally supplement service provision without adequate oversight or regulation by the government; and roles and responsibilities for service provision remain vague and un-coordinated. Rarely can any single entity address these problems on their own, thus, in some contexts, improving the quality or sustainability of public service requires purposeful and collective effort of a multitude of actors. Development practitioners are formulating ways to bring actors together to collectively address these problems-these are often referred to using a variety of terms, such as collaborative approaches, collective action approaches, and collective impact approaches. Designers and facilitators of these approaches can glean valuable lessons from the existing wealth of literature on collaborative work; however, this literature is largely built off experiences in high-income countries whose governments can more effectively provide, regulate, and/or outsource public services. The premise of this work is that key differences in government effectiveness, regulatory quality, the rule of law, political stability, voice and accountability, and cultural perspectives may limit the applicability of these lessons. Practitioners do not know which lessons from literature can directly apply and which may actually lead to failure.

Addressing these challenges requires a deeper understanding of: What factors (contextual conditions, design components, and intermediate results) contribute to the success of collaborative approaches for service provision in low-income countries? What is the relative importance of these factors, i.e., which are essential, and which are only important in some contexts? In addition to identifying factors, we also sought to investigate how these factors may differ because they are in a low-income country or in limited-governance contexts, as well as employed in service delivery applications. We sought to answer this through group consultation with experts on the subject, consisting of both practitioners and scholars with expertise in collaborative approaches in low-income countries, so as to expand upon the existing literature. Discussion and feedback were necessary to address inconsistencies and build a cohesive set of guidance; however, discussion introduces biases that influence decisions in group settings. The Delphi method [60-63] was selected to facilitate a panel of experts to discuss and prioritize key aspects of collaborative approaches for their application in low-income countries while also reducing group biases by allowing for anonymity over multiple rounds of rating with controlled feedback on panel opinions.

\section{Research Approach and Methods}

To develop a prioritized list of factors influencing the success of collaborative approaches for low-income country contexts, we first compiled a list of all relevant factors from literature and interviews. We then asked the expert panel to rate the importance of each factor over a multi-round, anonymous survey using the Delphi methodology $[63,64]$. 


\subsection{Compiling a List of Factors}

To compile a list of relevant factors, we reviewed literature and reports on collaborative approaches and conducted interviews with practitioners and experts. We searched the literature for approaches identified as "collective action" (e.g., [59]), "collective impact" (e.g., [10,55]), "interorganizational collaboration" (e.g., [40,65]), "collaborative management" (e.g., [45]), and "collaborative governance" (e.g., [66,67]). We searched the reference lists of the documents that resulted from this search, articles that cited them, and also reached out to our networks to request articles and reports related to the topic. Documents were excluded if they were about internally-focused collaboration, including collaboration between individuals or teams in a single organization, rather than collaborations that spanned agencies or organizations. We also excluded documents that focused at the community or village level, given that recent application of these approaches has focused on the sectoral level. While the information in these excluded contexts would be relevant, they may include different tactics and strategies when engaging multiple sets of individuals rather than organizations and government agencies. No documents were excluded based on publication date. We also conducted informal, semi-structured interviews in May-August 2019 with practitioners and experts. Interviewees included program directors, program officers, regional advisers, and implementation staff of international and local organizations focused on collaborative approaches for sustainable water and sanitation service delivery, experts in collective impact, and academics studying collaborative approaches more broadly. These interviewees were asked about key factors that have enabled or hindered progress, including contextual factors, as well as key decisions made during project design or implementation.

Using emergent coding methods in a web-based qualitative coding software [68], we analyzed the articles, reports, and interviews and identified a list of factors that have been found, or hypothesized, to lead to success in collaborative approaches. Qualitative analysis and review of additional sources were conducted iteratively until saturation, when no new factors were identified by reviewing any additional sources. This qualitative analysis [69] resulted in a list of 52 factors (Table 1), disaggregated into contextual conditions, design components, and intermediate results. Contextual conditions are existing factors inherent to the location, level of operation, and a regime that lie outside the control of project implementers and can only be influenced over long periods of time; these can influence planning stages of the approach. Design components are the steps, elements, or processes that are included by design within a coalition's functioning and activities; the project implementers have control over which design components are put in place. Intermediate results are key indicators or behaviors that the coalition exhibits over time; they are important milestones indicating progress toward success. This disaggregation is similar to how Ansell and Gash [18] and Emerson et al. [19] explain collaborative governance. 
Table 1. Factors that resulted from literature searches and consultation with experts. Factors added by panelists in Round 1 of the Delphi panel are noted with a "*”.

\begin{tabular}{|c|c|c|c|}
\hline Contextual Conditions & Design Components & & Intermediate Results \\
\hline $\begin{array}{ll}\text { 1. } & \text { Incentives or motivations to engage } \\
\text { 2. } & \text { Low turnover of positions } \\
\text { 3. } & \text { Compatible institutional priorities } \\
\text { 4. } & \text { Members know one another } \\
\text { 5. } & \text { Members respect each other } \\
\text { 6. } & \text { Members trust one another } \\
\text { 7. } & \text { Members capable to lead } \\
\text { 8. } & \text { Supportive local government } \\
\text { 9. } & \text { Relevant data is available } \\
\text { 10. } & \text { Members capable to engage } \\
\text { 11. } & \text { Interdependence of members * } \\
\text { 12. } & \text { Urgency for change * } \\
\text { 13. } & \text { Stable operating environment * } \\
\text { 14. } & \text { Unstable operating environment * }\end{array}$ & $\begin{array}{ll}\text { 1. } & \text { Rules and procedures } \\
\text { 2. } & \text { Focused on single level } \\
\text { 3. } & \text { Linked across multiple levels } \\
\text { 4. } & \text { Internal interaction and responsiveness } \\
\text { 5. } & \text { Centralized Leadership and facilitation } \\
\text { 6. } & \text { De-Centralized Leadership and facilitation } \\
\text { 7. } & \text { Visible Leadership and facilitation } \\
\text { 8. } & \text { Behind-the-Scenes Leadership } \\
& \text { and facilitation } \\
\text { 9. Neutral facilitation } \\
\text { 10. Facilitator pushes an agenda } \\
\text { 11. Externally-facing communication } \\
\text { 12. Progress measurement system accessed } \\
\text { 13. } \text { Py facilitator } \\
\text { 14. } \\
\text { 15. Cogress measurement system shared by } \\
\text { 16. Commont stakeholders represented } \\
17\end{array}$ & $\begin{array}{ll}\text { 18. } & \text { Common agenda } \\
\text { 19. } & \text { No hierarchy } \\
\text { 20. } & \text { Open membership } \\
21 . & \text { Closed membership } \\
22 . & \text { Engagement of technical } \\
& \text { government offices } \\
\text { 23. } & \text { Engagement of political officials } \\
\text { 24. } & \text { Facilitated by an insider } \\
25 . & \text { Funded by an insider } \\
\text { 26. } & \text { Voluntary participation } \\
\text { 27. } & \text { Mandatory participation } \\
28 . & \text { Transparent processes } \\
\text { 29. } & \text { Trust-building } \\
\text { 30. } & \text { Resources for administration } \\
\text { 31. } & \text { Regular meetings } \\
\text { 32. } & \text { Early wins } \\
\text { 33. } & \text { Shared decision making * }\end{array}$ & $\begin{array}{ll}\text { 1. } & \text { Legitimacy and credibility of the coalition } \\
\text { 2. } & \text { Shared ownership by members } \\
\text { 3. } & \text { Process continues between meetings } \\
\text { 4. } & \text { Senior-level representatives } \\
\text { 5. } & \text { Government leadership } \\
\text { 6. } & \text { Stable membership } \\
\text { 7. } & \text { Closely bonded group } \\
\text { 8. } & \text { Adaptation } \\
\text { 9. } & \text { Synchronized activities } \\
\text { 10. } & \text { Contributions are recognized * } \\
\text { 11. } & \text { Problems are solved * }\end{array}$ \\
\hline
\end{tabular}




\subsection{Delphi Expert Panel}

Using this list, we then employed a multi-round, anonymous survey with controlled feedback, the Delphi method [64], in an attempt to garner consensus on the importance of each factor specifically in low-income and weak governance contexts. A list of 35 experts was gathered based on authorship of reports or articles included in the review, as well as through recommendations by practitioners in our networks. We sought individuals with more than five years of experience establishing, facilitating, or studying collaborative approaches who had critically reflected on or evaluated the process in the format of reports, journal articles, book chapters, or books. We also targeted individuals with experience globally, but also invited experts whose work was solely in the United States, given that it is where most work on the topic has been completed.

Potential panelists filled out details about their experience, as well as the location, sector, and goals of up to five coalitions they worked in or with. This information was requested both as a check on the amount of experience a panelist claimed, as well as a filter to ensure the panel represented experience from low-income and weak governance contexts. Through this process, seventeen experts were selected to continue, all of which participated in all three rounds. Together their experience draws from over 70 coalitions spread out over 20 countries (Figure 1) and with four coalitions focused on global or trans-national activities. Of these, 29 coalitions were in the United States. Twelve of the seventeen panelists worked with at least one coalition in a low-income context. Conclusions from this study, therefore, are based on the collective wisdom and insights of experts with a vast amount of experience across the world, and thus, represent a robust consensus about the efficacy of various factors or characteristics influencing the success of collaborative approaches.

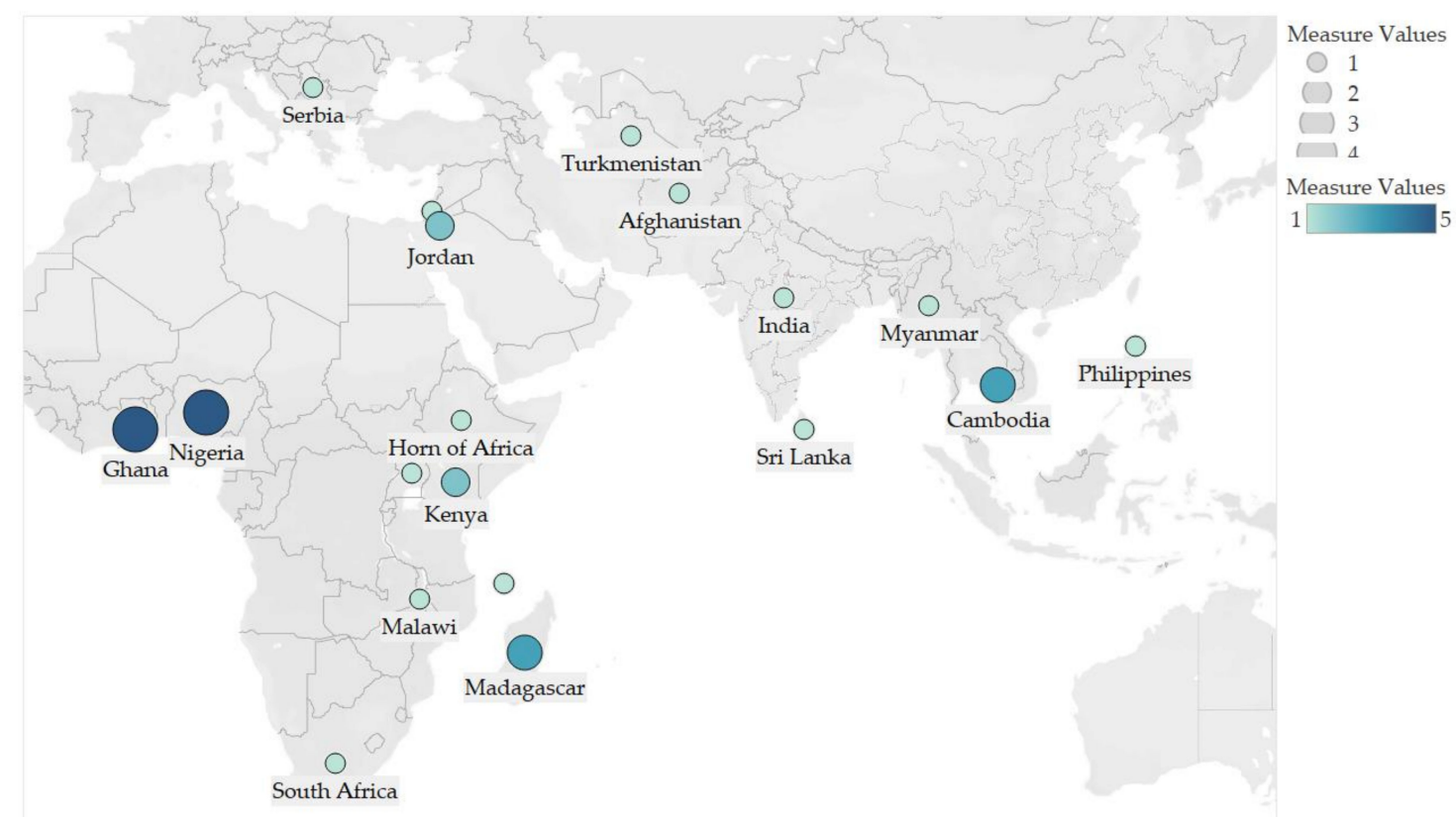

Figure 1. Fifty-one coalitions with which the panelists have worked, excluding the twenty-nine coalitions that took place in the United States. Bigger and darker shaded dots have greater numbers of coalitions in the country.

Panelists also were asked to identify which terminology they often use to refer to this type of collaborative approach. Three panelists identified this type of work specifically as inter-organizational collaboration, see Reference $[16,20]$, four as collaborative governance, see Reference $[18,66,67]$, four as collective impact, see Reference [10,57], one as collective action, see Reference [59], one as learning alliances, see Reference $[4,70,71]$, and one as networks. Three panelists mentioned wide range terms 
that they use to refer to this type of work, such as "Participatory methods, Coalition building, Collective action, Network building/development" or "collective action; collective impact; learning alliances; learning and practice alliances; movement; coalition" or "interorganizational relationships; collective action ... collective impact". The wide range of terminology speaks to the experience of the panel and emphasizes the need to connect these fields and standardize terminology.

Over a series of three rounds, the panel rated the importance of aspects of the implementation process for low-income country contexts-specifically, contextual conditions, design components, and intermediate results. We distributed the survey through Qualtrics (C). Experts were asked to rate each factor on a five-point scale of Not Important to Essential and were provided definitions for each rating and each factor. 'Importance' was selected for rating so that the process would result in a set of prioritized factors. No negative options were included given that the list only included items that had already been identified to be important in other studies; however, the option of "Not important" was defined to also include if a panelist believes that a factor is detrimental. An option of "Don't Know" was included in Round 1 so that confusion about any definitions could be clarified for future rounds; this option was removed starting in Round 2. In Round 1, panelists also had the opportunity to add any factors that they thought were missing. Panelists were invited to leave comments explaining their rating for each factor. After each round, we evaluated the rating distributions to determine which items had a significant level of agreement of importance by the panel, or 'reached a consensus'. The measures we used are described in the following section.

In Rounds 2 and 3, factors that did not reach a consensus were returned to the panel. Each panelist was given their score from the previous round, the median panel response, the distribution of panel responses, and selected comments from panelists. Selected comments reflected perspectives for each rating selected, so if the panel had ratings distributed across Of Moderate Importance, Very Important, and Essential, then representative comments for all three would be presented. Opposing viewpoints were also highlighted, when present. Panelist names were redacted so that, comments were kept anonymous. Panelists then re-rated the factors and were given the opportunity to submit any comments to justify their position. This method followed the standard Delphi process and recommendations from past studies $[60,64,72]$.

\subsubsection{Measuring Consensus}

In Delphi studies, there is no single established measure of dispersion used to determine consensus [73], and the measure selected can drastically change results [74]. Given the influence of this metric on results, we conducted a multi-step process to determine appropriate consensus measures for the study. Based on recommendations from von der Gracht [73], we calculated a series of measures of dispersion, such as mean, standard deviation, mean absolute deviation, median absolute deviation, interquartile range, coefficient of variation, and mean $+/-1.67^{*}$ standard deviation for each factor. We selected two measures of dispersion that best characterized consensus based upon the rating distributions and comments: A median absolute deviation of zero and a mean absolute deviation of less than 0.6 . These measures ensure that a factor will reach a consensus if (a) the majority of panelists select the same rating and (b) most 'minority' ratings are within one step $(+/-1)$ from the majority rating. The equations for these two measures are provided in Table 2.

Table 2. Measures of dispersion used for determining consensus.

\begin{tabular}{ccc}
\hline Measure of Dispersion & Equation, for a Set of Scores & Threshold Value for Consensus \\
& {$\left[\mathbf{X}_{\mathbf{1}}, \mathbf{X}_{\mathbf{2}}, \mathbf{X}_{\mathbf{3}}, \ldots \mathbf{X}_{\mathbf{n}}\right]$} & \\
\hline Median absolute deviation & median $\left(\mid X_{i}-\right.$ median $\left.\left(X_{1 \rightarrow n}\right) \mid\right)$ & 0 \\
Mean absolute deviation & mean $\left(\left|X_{i}-\operatorname{mean}\left(X_{1 \rightarrow n}\right)\right|\right)$ & $<0.6$ \\
\hline
\end{tabular}


For example, if nine panelists select a rating of 3, five selected a rating of 4 , and three select a rating of 2 , the median absolute deviation is 0 , and the mean absolute deviation is 0.52 . This factor would reach a consensus. Finally, as Round 1 allowed panelists to select a "Don't Know" option, a factor could only reach a consensus in Round 1 if it received zero "Don't Know" answers.

\subsubsection{Controls Against Bias}

Multiple steps were taken to reduce bias. During rating, questions were randomly ordered so as to eliminate bias introduced by the primacy effect, where questions displayed first are seen as more important, and contrast bias, where preceding questions influence interpretation of the current question [64]. Participants also were asked to provide reasoning for their rating, which minimized 'collective unconscious' bias, in which participants are guided by the median scores presented back. Presenting back reasoning that represents two opposing viewpoints helped reduce myside bias, in which participants fail to see the other perspective. Anonymity was maintained throughout the survey and in reporting results to eliminate influence from dominant or powerful figures. Experts had the chance to hear outlying views and change their original position and justify their own positions. Finally, median values are less subject to bias and to "rogue actors" than average values [73]. Thus, we calculated consensus using at least one measure of dispersion that is based around the median and also presented results in terms of the median panel response.

\subsubsection{Validity of Results}

We sought feedback from panelists by providing them with a written summary of results and access to an online interactive dashboard of results in January 2020, as well as by convening an online discussion using a webinar format in February 2020. We asked for feedback on both the Delphi panel process and the results, and we received positive feedback on both. In addition, we tested the consensus measures that were used to determine which factors reached a consensus, and thus, when factors could be taken out of subsequent rounds. To do this, we separated the 49 factors that reached a consensus from the nine that did not. We calculated Kendall's coefficient of concordance $(\mathrm{W})$, which assesses agreement among a panel of raters on a scale of 0 (no agreement) to 1 (complete agreement) [73]. Scores greater than 0.5 are considered 'good' agreement [75]. The set of factors that reached a consensus had a score reflecting good agreement $(\mathrm{W}=0.523, p<0.01)$, while the factors that did not reach a consensus had a score reflecting moderate agreement $(W=0.401, p<0.01)$ [73]. In addition, agreement increased over each Round, increasing from 0.347 in Round $1(p<0.01)$ to 0.461 in Round $2(p<0.01)$ and 0.532 in Round $3(p<0.01)$. This test confirms that the consensus measures we used decently reflected panel agreement.

\section{Results}

In Round 1, the panel reached a consensus on $21 \%$ of the 52 factors that were rated, and also added seven factors, bringing the total number of factors rated to 59 . The seven added factors are denoted by a " ** in Table 1. By Round 2, the panel had reached a consensus on $50 \%$ of the factors, and by Round 3, $84 \%$ of factors reached a consensus. Thus, by the end of three rounds, the panel agreed on the importance of 49 factors, but disagreed on the importance of nine factors. Eight factors were agreed to be Essential for success, while the others ranged from Of Little Importance to Very Important. A complete table of results with factor definitions, median scores, distributions of responses, and comments is provided in Supplementary Information, Tables S1-S3, as well as a link to an interactive dashboard of all results. Rich qualitative data from open-ended questions and discussions supplement the ratings to reveal unique insights into gaps in the literature. This section reviews the quantitative results, including the median ratings and factors that did not reach a consensus, followed by trends revealed by the qualitative analysis. A summary of key considerations for practice and future research that resulted from this work can be found in Appendix A, Table A1. 


\subsection{Contextual Conditions}

Contextual conditions are existing factors inherent to the location, level of operation, and a regime that lie outside the control of project implementers and can only be influenced over long periods of time; these can influence planning stages of the approach. The panel agreed that two contextual conditions are Essential, meaning they must be present for a collaborative approach to succeed: That members have both (a) incentives or motivations to engage in the coalition; and the (b) skills to ultimately take on leadership roles, where "if someone becomes a leader because of the given collaborative context, then it is crucial that the person has the right skills and resources to lead well" (Figure 2). Other contextual conditions were seen as Very Important, where a coalition would likely not succeed if it were not present, including compatible institutional priorities and supportive local government. These factors, though important, fell short of Essential either because there are strategies for moving forward even if they are not in place or because they can be Essential in most contexts, but not all. The panel also agreed that, while respect and trust between members are less important at the onset, they become essential later on in the process. Definitions and final ratings for all contextual conditions can be found in Supplementary Information Table S1. All contextual conditions except for one, unstable operating environment, reached a consensus. 'Unstable operating environment' was added by a panelist in the open-ended section of Round 1, and in the subsequent rounds, panelists could not agree on whether it is enabling or hindering. Some felt that instability could severely hinder the ability of the group to make progress, as one panelist explains:

"When faced with highly unstable circumstances, many actors revert to coordinating activities instead of more integrated collaboration. If a coalition is built during a time of greater stability, then those actors deeply engaged in a coalition can respond better to moments of crisis or instability when they arise. Expecting to build high levels of trust and collaboration in the midst of a crisis without having previous collaboration among actors presents more challenges than if there is a history of engagement".

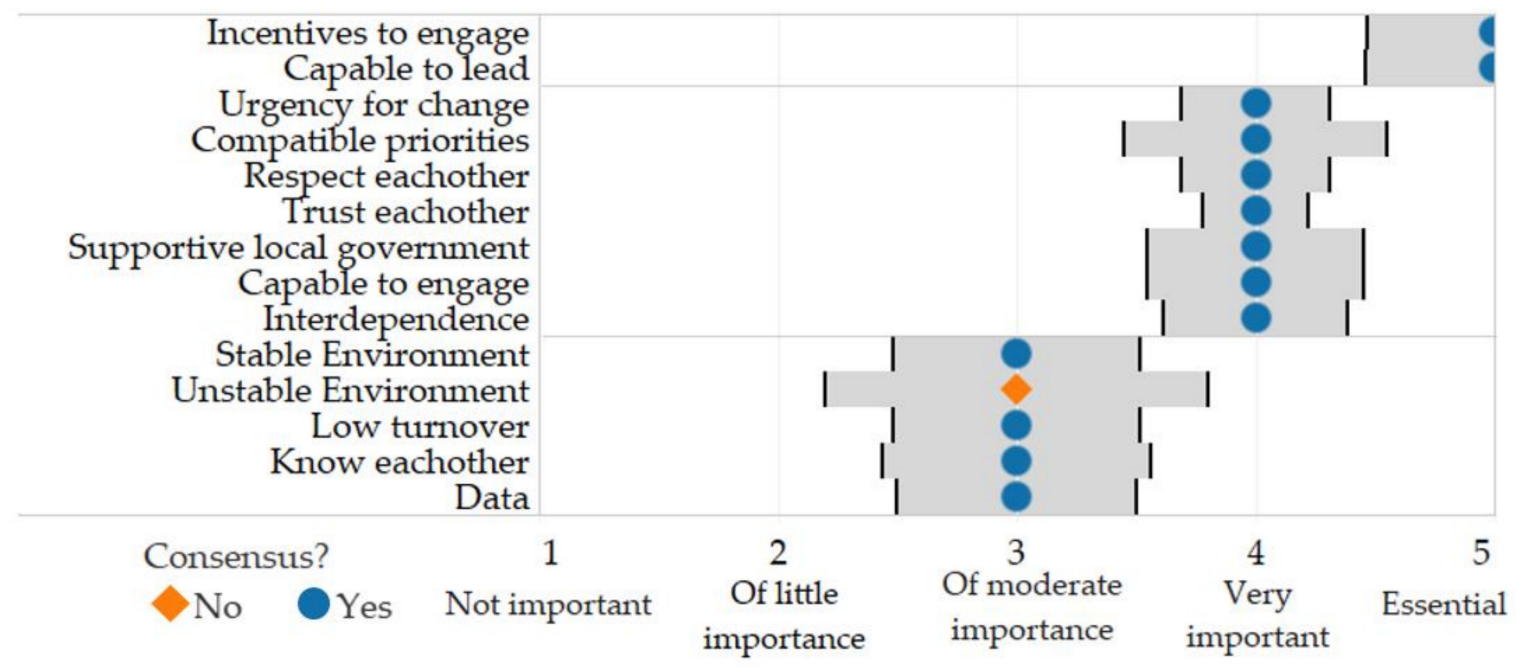

Figure 2. Final scores for all contextual conditions, ordered from highest to lowest ratings. Dots show the median score. Gray bars show the mean absolute deviation of responses. Blue dots are factors that reached a consensus, and orange diamonds did not reach a consensus.

However, others saw it as enabling, where instability can be an impetus or catalyst for collaboration because of the need to bring people together. Another panelist explains this other perspective: 
"The instability may impact the ability to concretely plan for and achieve intended outcomes, but [it] is critical for partners to work together on common challenges in an unstable context... By coming together to tackle common challenges, the coalition may have opportunity to impact the stability of [the] enabling environment. It may be necessary to explore different models for collaboration in stable versus unstable contexts... for better development outcomes".

Instability of political and institutional environments appears to play a role as both an impetus and a hurdle for collaborative approaches. In so, it influences decisions about what level of collaboration is required.

\subsection{Design Components}

Design components are the steps or elements that are included by design within a coalition's processes and activities. The panel agreed on four design components that are Essential for success, meaning that they must be implemented so that the coalition succeeds: That actions and decisions are transparent, that all relevant stakeholders are represented, and that the group agrees on a common vision and a common problem (Figure 3). Definitions and final ratings for all design components can be found in Supplementary Information Table S2. Transparency and common vision easily reached a consensus in Round 1 with few comments from the panel. Having all relevant stakeholders represented is quite difficult to achieve, which caused some panelists to rate this as Very Important, rather than Essential. Some panelists advised for diversity without overrepresenting a single sector, interest, or area of expertise; others argued that too much diversity could lead to a lack of alignment and create more problems. Figuring out the right balance, however, is Essential. The discussion on Common problem included some important caveats, even though it too reached a consensus on Essential. After some discussion, the panel came to the consensus that not all facets of the problem need to be agreed upon and different actors can be working on different aspects of the problem or on compatible problems.

The panel agreed that whether or not the coalition was funded by an insider was Of Little Importance, meaning that it rarely contributes to success. This is particularly relevant as often in development work, and collaborative approaches are funded by international NGOs or donors. This was the only factor that received this low rating and reached a consensus-the factor having the facilitator push an agenda resulted in a median of Of Little Importance as well, but the panel largely disagreed about it. One panelist brought up that if no one pushes an agenda, then nothing can get done, while others argued that a core tenant of facilitation was to be neutral. Six other design components did not reach a consensus. A diversity of viewpoints existed on the importance of having no hierarchy, for the facilitator to be an insider, and if the group should operate at multiple linked levels, although the median centered on Very Important. The panel also disagreed on whether or not membership should be closed, leadership should be centralized, and for the measurement system for tracking progress should only be accessed by the facilitator- these factors centered on a median of Of Moderate Importance.

Hierarchy between participants of a collaborative group was a key differentiator discussed in the introduction to this article. While most collaborative approaches have been developed and studied in cultures with low power 'distance', where individuals tend to consider all members as equal, many low-income contexts have cultures that expect and accept large differences in power between individuals $[25,26]$. Most panelists viewed having no hierarchy as Essential or Very Important, but a few strong viewpoints emerged that argued against it. They claimed that there are always power imbalances and "the claim or idea that collaboration replaces hierarchy and that all players come to the table as equal is not only silly, it is dangerous"-arguing that power imbalances should be acknowledged and leveraged rather than seen as bad. In response, other panelists argued that even if there are formal hierarchies and power differences, members should all "feel they are equal", especially in fields with a lot of competition. This feeling of no hierarchy creates an environment where members will express their interests without being overpowered. While the panel did not come to a consensus 
on this item, the median value was Very Important, indicating that this is an important factor that deserves further attention in these contexts.

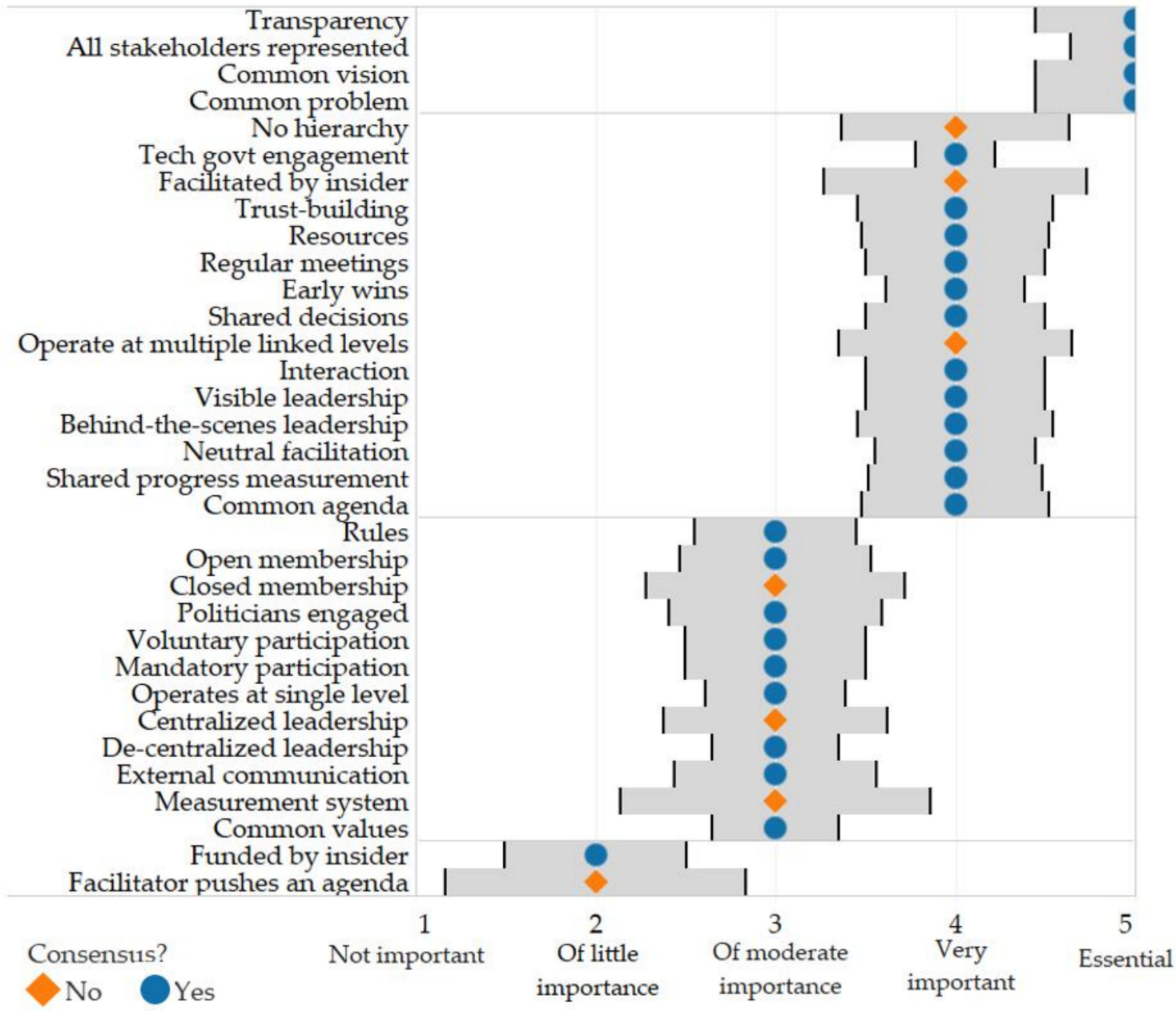

Figure 3. Final scores for all design components, ordered from highest to lowest ratings. Dots show the median score. Gray bars show the mean absolute deviation of responses. Dark blue dots are factors that reached a consensus, and orange diamonds did not reach a consensus.

\subsection{Intermediate Results}

Intermediate results are accomplishments or behaviors that a coalition aims to achieve as it works towards a common vision. The panel agreed that two intermediate results are Essential, meaning that without these steps the coalition will not reach success: (a) Legitimacy and credibility of the coalition and (b) adaptation (Figure 4). While few comments were provided for either of these intermediate results rated as Essential, panelists' comments for other factors stated how specific design components could help build legitimacy and credibility. Having a credible leader and facilitator is very important, which some panelists agreed could be achieved by having an 'insider' serve as the leader or facilitator. This is not always the case, as one panelist indicated: "Sometimes you need an insider to give you legitimacy and sometimes an insider undermines legitimacy". One panelist pointed out that it is "Much better if it is [funded by an insider], but [it] can be funded by outsider. Also note that there is some equilibrium between the facilitator and funder. If the funder is not an insider, it makes it more essential for the facilitator to be". Given that the panel agreed that it is an Essential intermediate result, implementers should frequently assess legitimacy, and perhaps use it as a leading indicator for success. 


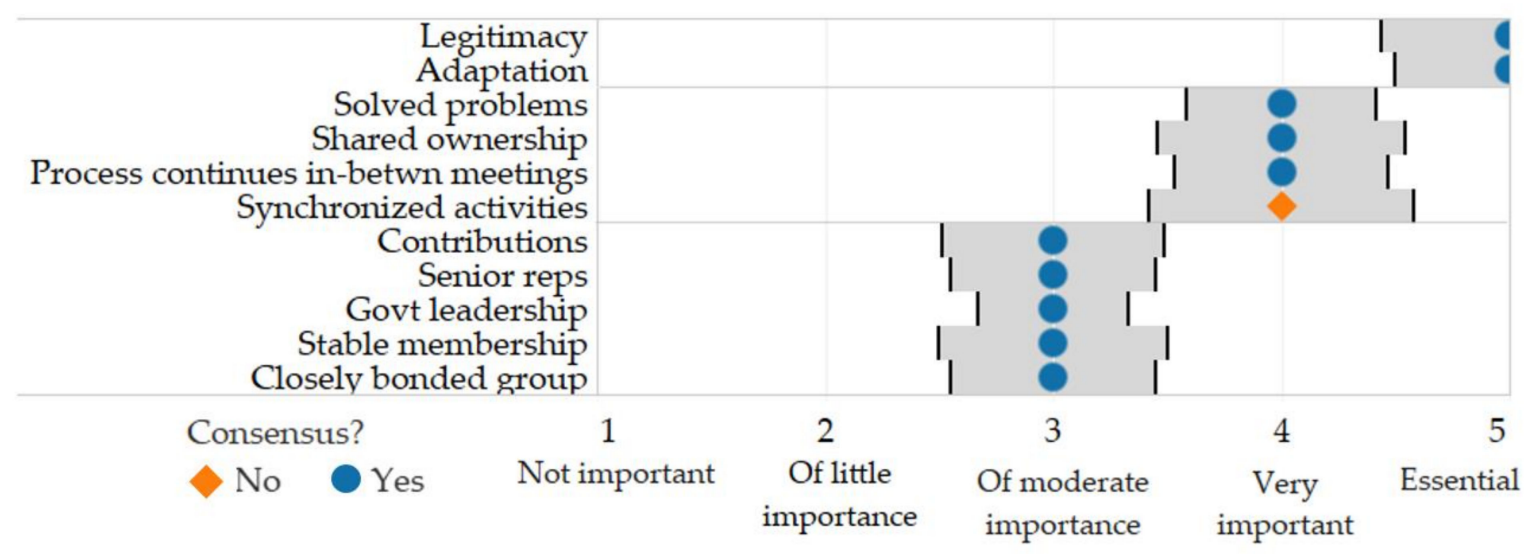

Figure 4. Final scores for all intermediate results, ordered from highest to lowest ratings. Dots show the median score. Gray bars show the mean absolute deviation of responses. Blue dots are factors that reached a consensus, and orange diamonds did not reach a consensus.

The panel also agreed on three intermediate results that are Very Important, meaning that the coalition will likely not reach its ultimate outcomes if the results are not observed: Problems are solved, shared ownership, and the process continues between meetings. All of these intermediate results except for one, synchronized activities, reached a consensus. Synchronizing activities was a controversial design component because panelists disagreed about how highly coordinated or synchronized activities need to be. One panelist argued: "There should be a strong focus on getting to a synchronized or integrated set of actions/activities that wok towards achievement of the coalition's goal". However, many panelists saw the word "synchronized" as too strong - that instead of synchronizing all activities, members should coordinate activities. Others argued one step further: That too much coordination could be harmful, and some redundant activities may be beneficial. The differences in perspectives appear to reflect different levels of intensity of collaboration. Definitions and final ratings for all intermediate results can be found in Supplementary Information Table S3.

\subsection{Special Focus: Government Engagement}

Government engagement was one of the more contested topics discussed by the panel. The panelists largely divided into two groups while rating the importance of having a supportive local government (contextual condition), engaging technical and/or political government officials in the process (design components), and having government leadership as an end-goal (intermediate result). Despite disagreeing viewpoints, all four factors reached a consensus. The distribution of responses for each is shown in Figure 5.

The first group took the cautious stance that if/when/how to engage government was dependent on the objectives of the coalition and on the context-for example, if an objective was advocacy then it could not be led by the government, or if the government is weak or unstable it may be dangerous to rely too much on their support. They further clarify that it matters when (and for what) the government is involved and that "the government must be trustworthy, in and of itself, to work; especially since we're talking about impoverished places in the world". The panel noted that success often has to be demonstrated first before the government will get involved and that changing governments and political climates can shift support over time. Too much reliance on the government may be harmful: "It's a dangerous thing to rely 'too much' on the power of government leaders'/politicians' support to make things happen ... they the coalition should also feel empowered to tackle the issue at hand *despite* the lack of support before/at the beginning of their collaboration". 

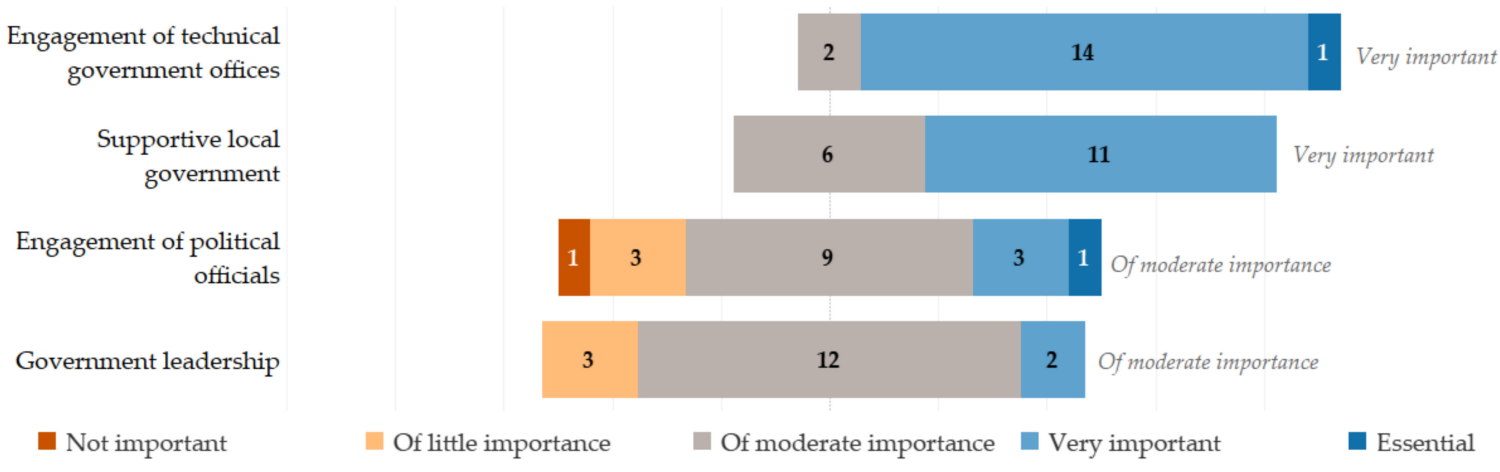

Figure 5. Distribution of final ratings for four factors related to government. All four reached a consensus. Final median ratings are noted next to each factor.

The other group argued that adequate government engagement is essential for scale, sustainability, and self-reliance development objectives, though the most appropriate way to engage government can vary: "Forty-plus years of development experience and we still have to learn this?... Show me a successful large-scale change movement or coalition that has not engaged politicians. This seems so obvious to me that I almost don't understand the question". In response to warnings from other panelists regarding the political and volatile nature of involving government, one panelist argued that the strategic aspects of involving government outweigh any political risks:

"It's important to look beyond the political, to the technical and strategic development aspects of government. Building this type of engagement or engaging at [these] levels provides [a] structural basis to anchor approaches and efforts. As such the local government support is very important, particularly, where strategically engaged to achieve common/tandem objectives and goals, that align with development or strategic plans".

One panelist in the latter group pointed out that in low-income communities with "cultural and power dynamics that might revere local leaders or alternatively fear authoritarian leaders, this will be important throughout the process. Moreover, especially if project leadership and funds come from outside the community". This group generally felt that there may be a few unique situations where the government need not directly engage in the process, but at the very least, government consent is absolutely required. If consent is not obtained, one panelist explained, then the government can feel threatened, and if there is any "perceived threat, offense, or 'illegal' behavior of participants in the collective work... the government support can quickly shift to adversarial and outright undercutting/banning of the collaborative work".

This difference in perspectives largely revolved around whether "engagement" meant that government should be kept informed and updated versus being actively involved. For example: "We have found that platforms that function independently of government, but include them as 'observers' (as a way of coordinating and sharing information/to maintain support) to be more effective than those with government as a member". Another panelist argued that engaging technical government officials is Very Important, while involving political officials is Of Moderate Importance: "I still believe that engaging political officials is of moderate importance as the technical government offices can do their part in influencing other critical actors within the government. In addition, in many cases, it is the technical offices that help make the case for adopting certain practices or activities internally and can be important advocates". One panelist pointed out that they would rate the importance of engaging political officials higher if "engaging simply means keeping elected officials informed and updated on a regular basis", but "to me, engaging means involving them in meetings, discussions, decision-making processes, and overall collaborative approaches before arriving at any solutions". 
Practitioners and scholars alike would benefit from a deeper investigation into how to involve or engage political and technical government officials and at what stages of the process their involvement, engagement, and/or leadership is most important.

\subsection{Special Focus: Common Orientation Around an Issue}

The literature on collaborative approaches always acknowledges the importance of the group reaching a common orientation around an issue. However, in doing so, literature often interchangeably cites the importance of agreeing on a common vision (i.e., a long-term mission or goal), key problems limiting that vision, a set of activities to address those problems in a common agenda or workplan, and a set of common values that the group uses to carry out that agenda (Figure 6). These can be visualized using a hypothetical problem tree, with a vision being the highest and most broad level, followed by a series of problems that may be affecting or limiting the vision. Then, each problem could have a series of actions or activities that seek to address it, forming a common agenda.

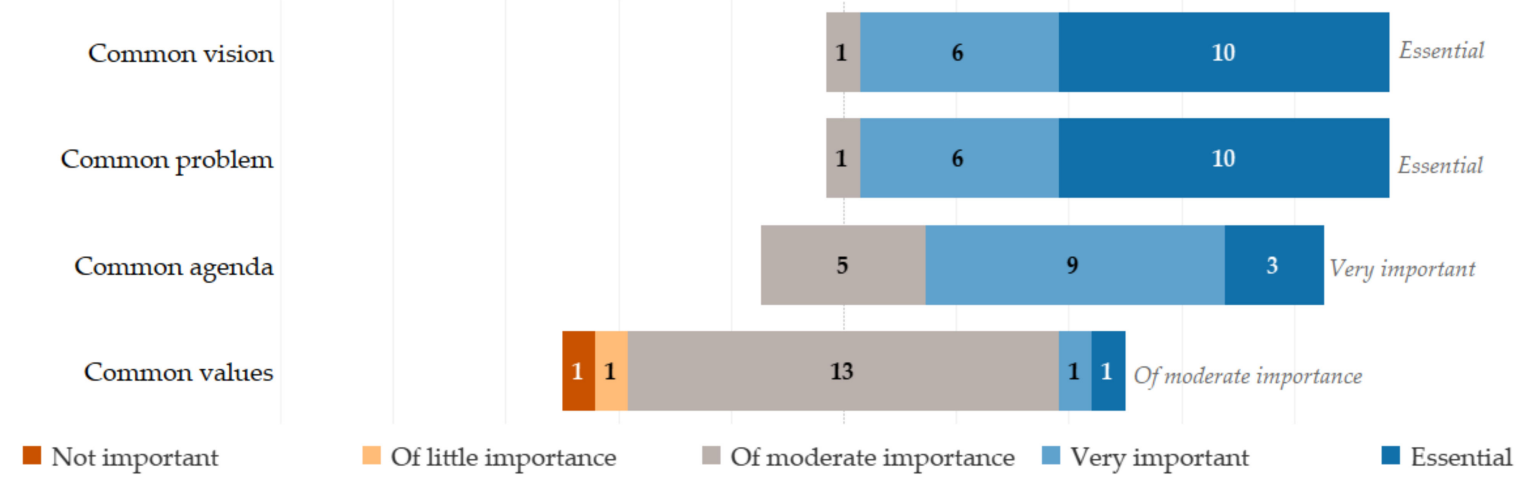

Figure 6. Distribution of final ratings for four factors related to agreeing on a common orientation to the issue. All four reached a consensus. Final median ratings are noted next to each factor.

Each additional level takes significantly more resources and time to reach an agreement than the prior, so it is useful to disaggregate these and figure out which level of agreement is most important.

The panel agreed that it is Essential to have a common vision and a common problem, Very Important to agree on a common agenda, and Of Moderate Importance to agree on a set of common values. After discussion, the majority of panelists came to a consensus that agreeing on a common problem was Essential, noting that not all facets of the problem need to be agreed upon, and different actors can be working on different aspects of the problem or on compatible problems. The panel also agreed that the common agenda, or on the roadmap to solve the problem, was Very Important. Notably, one panelist explained this common agenda is less important than agreeing on a problem and vision because sometimes "too much effort is placed in generating a common agenda versus allowing time for coalition members to test approaches, build relationships, and generate evidence together in the early stages of a coalition's existence". Common values were seen as potentially helpful but could differ amongst participants and not affect the achievement of a goal. If values were too similar amongst the participants, panelists felt it could "potentially lead to the dangers of groupthink" and that "insisting on everyone thinking the same way or working in the same way from the get-go kills innovation". In the end, panelists were satisfied with a rating 'Of Moderate Importance' alongside the guidance of having a process for negotiating differences and having a few values to generate buy-in. This disaggregation revealed that agreement on a common vision and problem should receive the most attention as they are Essential for success. 
In international development, a common vision is often dictated by the United Nations and other global agencies. For example, universal access to clean water and basic sanitation services for all by 2030 is a standard goal set by the UN Sustainable Development Goal 6 (SDG 6). In collaborative approaches in the water and sanitation sectors, many actors have a vision that reflects this ambitious target, but many actors prioritize different problems that must be addressed to reach it. For example, some may focus all efforts on infrastructure expansion without ensuring that the local government has the spare parts or resources to maintain water pumps or empty latrines—or even ensuring that responsibilities for maintenance are transferred. Using this example, to get a group of these actors to begin to collaborate on improving water or sanitation services locally, it will be essential to move beyond just the common vision of SDG 6 and reach agreement on which problems the group will seek to tackle. Future work should consider this disaggregation and clearly define which level of agreement the group reached.

\section{Discussion}

This study was motivated by a need to understand not only which factors influence the design, implementation, and monitoring of collaborative approaches in low-income country contexts, but also to identify their relative importance to one another. In addition to this, we also sought to broadly investigate how these factors may be unique both to limited-governance contexts and to service delivery applications. We investigated these differences through a virtual webinar discussion with the panelists that we convened in February 2020, supplemented by a qualitative analysis of panelist comments. Through this process, another difference emerged related to the level of intensity of collaboration that the implementers desire to achieve. These differences are discussed in the following sections.

\subsection{Differences in Low-Income Country Contexts}

The major differences that the panelists identified as unique to low-income country contexts are the role and influence of donors, designing for stable versus unstable political and institutional environments, acknowledging and working around a lack of data, accommodating inevitable high turnover rates and inadequate knowledge transfer, and building leadership skills of members so that they can take on leadership roles. For example, one panelist explained the role of collaborative approaches in highly unstable environments:

"Instability provides a greater impetus for collaboration as different strengths, skills and perspectives are brought to bear on a common problem/challenge. The instability may impact the ability to concretely plan for and achieve intended outcomes, but is critical for partners to work together on common challenges in an unstable context. It may be necessary to explore different models for collaboration in stable versus unstable contexts, to explore capacity for better development outcomes".

Additional quotes for each factor unique to low-income country contexts can be found in Supplementary Information Table S4. Additionally, the panelists pointed out that how some factors need to be implemented or established may differ in low-income country contexts. These include how to generate legitimacy, how to structure leadership and facilitation, how to deal with power imbalances, and how to best involve or engage political and technical government officials. Quotes from panelists to support each of these factors are also in Supplementary Information Table S4. As an example, one panelist emphasized how much leadership and facilitation structures depend on the culture and the context:

"Culturally, is this a patriarchy where centralized decision making makes sense and feels 'normal' for the constituents? Is there so much turbulence that the locals would much prefer some clear accountability through quasi-bureaucratic structures that come with centralized decision making? Alternately, is this a collective society where participation is the norm?" 
Broadly, we recommend that these differing factors should all be considered by funders and implementers of collaborative approaches in low-income country contexts. Political economy analyses and other institutional strength assessments may be useful tools for implementers to understand these differing factors. Simultaneously, funders of development programs and projects should review proposals for a comprehensive understanding of these differing factors.

\subsection{Differences in Service Delivery Applications}

We also investigated which factors may be different when designing, implementing, and monitoring collaborative approaches that are applied to strengthen service delivery, for example, water, sanitation, energy, and transportation services, as opposed to other applications, such as advocacy, gender equity, human rights, or entrepreneurship work. Key differences that emerged include government engagement and agreed-upon shared metrics.

Regarding government engagement, one panelist pointed out the engagement of politicians differs by application: "There are cases where there is a need to organize advocacy campaigns to influence policy and which may be against existing policies. In these cases, there may need to be determined spaces or facilitation mechanisms to engage with elected or political officials". For non-service delivery applications, such as advocacy, government engagement in collaborative approaches may be less critical. Considering the mandates of local governments to provide public services; however, collaborative approaches that intend to strengthen service delivery must involve governments as they hold the mandate and make the decisions regarding how services are delivered.

Another key difference specifically in the delivery of water, sanitation, energy and transportation services may be the sector-wide acceptance of a set of agreed-upon common metrics revolving around universal access. These sectors do not suffer from sector-wide disagreements about how to measure the success of advocacy, gender equity, peacekeeping, or conservation programs. Related to collaborative approaches, this influences the contextual condition of having compatible institutional priorities and the design component of achieving common orientation around an issue, which may require less negotiation overall.

\subsection{Differences in Intensity of Desired Collaboration}

Finally, this investigation also revealed another difference in factors: How 'collaborative' the collaborative approach intends to be. A few times during the panel discussion, the reason that experts disagreed could have been because they were picturing a more intense form of collaboration than others. For example, when discussing stable operating environments, one panelist explained how:

"When faced with highly unstable circumstances, many actors revert to coordinating activities instead of more integrated collaboration. If a coalition is built during a time of greater stability, then those actors deeply engaged in a coalition can respond better to moments of crisis or instability when they arise. Expecting to build high levels of trust and collaboration in the midst of a crisis without having previous collaboration among actors presents more challenges than if there is a history of engagement".

This theme also emerged related to the intermediate result of synchronized activities, where many panelists felt that the word "synchronized" was too strong-noting that instead of synchronizing all activities, members should coordinate activities. Some panelists argued that some complementary or redundant activities may be beneficial. Others, however, argued for the design of synchronized or complementary or "mutually reinforcing" activities. The two panelists who settled on Essential for Round 3 are experts in the collective impact approach, which specifically calls for "mutually reinforcing activities" [10]. The differences in perspectives appear to reflect different levels of intensity of collaboration, where highly coordinated complementary synchronized activities may be very important or essential for very intensive forms of collaboration, such as collective impact. For example, highly integrated forms of collaboration may be needed when a coalition desires to change 
deeply-rooted roles and responsibilities, regulations, or funding mechanisms. For less intensive forms of collaboration, such as for improving information flow and reducing duplication of efforts amongst interdependent actors in a system, it is enough to just coordinate some activities and allow for some redundancies in activities. However, different levels of intensity of collaboration are not widely prevalent in the literature on collaborative approaches.

Fortunately, there has been a lot of recent work that has started to identify and disaggregate approaches into different types of collaboration, for example into Informative, Consultative, Collaborative, and Integrative [59]; Networking, Cooperation, Coordination, Coalition, and Collaboration [76]; Consultation, Contracting, Commissioning, Co-production, Co-management, and Certification [12]; Information, Consultation, Involvement, Collaboration, and Empowerment [48]; Collaboration vs Consultation [77], and "Samhandling" which signifies interaction, collaboration, cooperation, and coordination in a single word [53]. While this a good first step, none of these efforts appears to build on one another or attempt to synchronize terms or definitions. Without a concerted effort to standardize definitions or typologies, scholars will continue to talk past one another and miss many opportunities to compare their work with one another.

\subsection{Future Work}

Overall, there is an overwhelming need for future studies to investigate factors that influence collaborative approaches for service delivery, in low-income countries, and at different levels of intensity of collaboration. A summary of key considerations for future practice and for future studies is provided in Appendix A, Table A1. These studies should consider factors that influence program design, implementation, and monitoring, including key contextual conditions, design components, and intermediate results. As this expert panel has shown, there also a need for future work to not only identify factors, but also investigate their relative importance to one another, such as which are necessary, but not sufficient, conditions for success. Framing future work in this way can start to identify which factors may always be essential versus which may only be essential in some contexts and where other factors are present. This type of work is highly context-specific, as demonstrated by the fact that it took discussion and reflection over multiple rounds to agree on what factors are essential. While there is certainly no single set of factors that always lead to the success of collaborative approaches, the results highlight what factors may be more important than others. This simplified list of factors and results, rated by importance, can guide program development and monitoring for both implementers and funders.

In addition, future work should seek to connect the multiple bodies of literature on collaborative approaches. The diversity of perspectives seen in the panelist discussions and the fact that panelists only agreed on the importance of roughly a fifth of the items in the first round of this expert panel speaks to this need for further cross-discipline research and dialogue.

\subsection{Limitations}

Studying collaborative work does not exist without limitations. We are attempting to parse and segment an immense list of context-dependent considerations for collaborative approaches into tidy lists of factors. Some will argue that this perspective is overly structured and reductionist, that there is no 'periodic table of elements' for collaborative approaches, and that collaboration is a process consisting of interactions between individuals that build on one another. However, we did this as we believe it is the most useful way to communicate the vast amount of information and advice to development practitioners. We are not seeking to establish one unified or universal language amongst the literature. Further, every case can consider 'success' differently. We defined success as the coalition making progress toward the vision it set out to achieve, but we acknowledge that differing expectations and goals exist. A core assumption of this work is that limited-governance contexts pose unique challenges that require some additional considerations, but these considerations may also apply to 'stable' governments that still have corruption, are highly political, and are skeptical of collaboration. 
Another limitation is that in an attempt to provide a broad basis of guidance, we consulted with experts who have experience in many contexts, but not all. Despite these limitations, we believe that this study adequately represents the perspectives of a diversity of experts on collaborative approaches in low-income countries, which has not been presented in the literature before.

\section{Conclusions}

This paper presents the results of an expert panel that was convened to rate the importance of factors found to contribute to the success of collaborative approaches in low-income countries. The panel was comprised of seventeen experts with experience in over 70 coalitions working in over 20 low- and middle- income countries, as well as in global or transnational coalitions. Thus, the results represent a vast amount of experience across the world on the efficacy of various factors or characteristics influencing the success of collaborative approaches.

Collaborative approaches are increasingly being implemented in low-income countries to improve service delivery because these contexts often pose problems that are too complex for any single actor to solve on their own. However, the wealth of literature on collaborative approaches has been based on experiences in high-income countries with strong governance regimes and clear mandates for service provision. To further complicate the lack of evidence, existing literature uses a wide range of terms to define collaborative approaches, as well as their core tenants. These gaps challenge organizations or agencies seeking advice to set up their approach "the right way", for funding agencies who may desire to fund these approaches but cannot find adequate evidence or metrics to use to monitor success, and for researchers seeking to compare existing approaches to the wider body of literature. Inconsistencies in terminology also challenge the ability for implementers to build legitimacy with local stakeholders, service users, and governments. To begin to fill these gaps, we consulted with experts from both academia and practice to come together and provide concrete guidance to the development sector regarding the key factors that influence collaborative approaches for service delivery in limited-governance contexts, such as low-income countries.

The panel rated a total of 58 factors that have been found to contribute to the success of collaborative approaches-divided into contextual conditions, design components, and intermediate results. The panel rated all factors for their relative importance in low-income country contexts and service delivery applications. The panel reached a consensus on eight factors that are essential for success: When planning a collaborative approach, potential members must (1) have incentives and motivations to engage and (2) be capable of leading; the approach must be designed so that (3) it has transparent processes, (4) it has all stakeholders represented, and (5) members agree on both a common vision and (6) a common problem that limits that vision; and finally, accomplishments that must be achieved include the group being (7) seen as legitimate and credible, and (8) able to adapt during the process. The final ratings of all 58 factors provide valuable insights into their relative priority for development practitioners by parsing out which factors are necessary for all contexts versus which only may be necessary for some contexts. For example, the panel agreed that government leadership is Of Moderate Importance, meaning that, while in some cases government leadership is not necessary for success, in other cases it plays an important role in success, such as in cases with a goal of promoting government self-reliance.

In this study, we also investigated how factors may be unique to limited-governance contexts and to service delivery applications. The panelists pointed out that factors unique to low-income country contexts are the role and influence of donors, designing for stable versus unstable political and institutional environments, acknowledging and working around a lack of data, accommodating inevitable high turnover rates and inadequate knowledge transfer, and building leadership skills of members so that they can take on leadership roles. Other factors differ in how they need to be implemented in these limited-governance contexts, including how to generate legitimacy, how to structure leadership and facilitation, how to deal with power imbalances, and how to best involve or 
engage political and technical government officials. Factors unique to service delivery applications include government engagement and agreeing upon shared metrics.

Thus, this study contributes to the broad base of literature on collaborative approaches by calling out key differences that emerge when applying collaborative approaches to service delivery applications and to limited-governance contexts. This responds to calls from past literature to further investigate the enabling and hindering factors of successful collaborative efforts in low-income countries [48,78]. In addition, past studies and frameworks for various collaborative approaches advocated for numerous factors that should be considered in design; however, they did not clarify the relative necessity or importance of each relative to others. This study facilitated a panel of experts to explicitly rate the importance of over fifty factors and showed that there is a wide diversity in factor importance. This study also adds value by organizing, coalescing, and drawing connections between the plethora of literature and terms related to collaborative work.

This study also contributes to practice more broadly, as the list of factors and their ratings of relative importance can provide guidance for designers (see Appendix A, Table A1) of collaborative approaches for service delivery in limited-governance contexts, such as low-income countries, for which guidance does not explicitly exist. Any collaborative approach being proposed should clearly understand the factors that uniquely affect their approach design in limited-governance contexts and/or for service delivery applications, and funders should evaluate proposals for this understanding.

Supplementary Materials: The following are available online at http://www.mdpi.com/2071-1050/12/7/2612/s1, Table S1. Contextual Conditions definitions and final results, Table S2. Design Components definitions and final results, Table S3. Intermediate results definitions and final results, Table S4. Factors unique to low income country contexts, with supporting quotes from panelists.

Author Contributions: Conceptualization, K.P., A.J.-W., and S.P.; methodology, K.P., A.J.-W., M.K.; software, K.P.; validation, K.P., A.J.-W., and M.K.; formal analysis, K.P.; investigation, K.P., A.J.-W., S.P., and M.K.; resources, A.J.-W., K.L.; data curation, K.P.; writing—original draft preparation, K.P., A.J.-W.; writing-review and editing, K.P., A.J.-W., M.K., S.P., and K.L.; visualization, K.P.; supervision, K.L., A.J.-W., S.P.; project administration, K.L., A.J.-W.; funding acquisition, K.L., A.J.-W. All authors have read and agreed to the published version of the manuscript.

Funding: This work was completed with financial support from the Sustainable WASH Systems Learning Partnership through USAID under the terms of the Cooperative Agreement AID-OAA-A-16-00075 to the University of Colorado Boulder. The contents are the responsibility of the University of Colorado Boulder Sustainable WASH Systems Learning Partnership and do not necessarily reflect the views of USAID or the United States Government. For more information, visit www.globalwaters.org/SWS. Data collection methods used in this research were reviewed by the University of Colorado Boulder under IRB Protocol 19-0207.

Acknowledgments: The authors deeply appreciate the time and thought that all panelists contributed to this study, who will remain anonymous. In addition, the authors would like to thank the anonymous peer reviewers, whose reviews greatly strengthened this article, and the editor of this special issue, who pulled together this issue on such a pertinent and timely topic. The authors also would like to thank the interviewees and advisors who helped build out the list of factors: Adeeb Mahmud, Tjip Walker, Jonathan Annis, John Butterworth, Angela Huston, Marieke Adank, Muhammed Ibrahim, Lucia Henry, Rob Hope, Jeremy Sokulsky, and Neera Singh. The first author also would like to thank Jessica Pugel for her support on the validation statistics for the consensus measures. Publication of this article was funded by the University of Colorado Boulder Libraries Open Access Fund. The data for this paper were collected using Qualtrics software. Copyright (C) 2020 Qualtrics. Data visualizations were created using Tableau software version 2019.4.2. Copyright (C 2020 Tableau Software.

Conflicts of Interest: The authors declare no conflict of interest. 


\section{Appendix A. Summary of Considerations for Future Practice and Research}

Table A1. Summary of considerations for future practice and research.

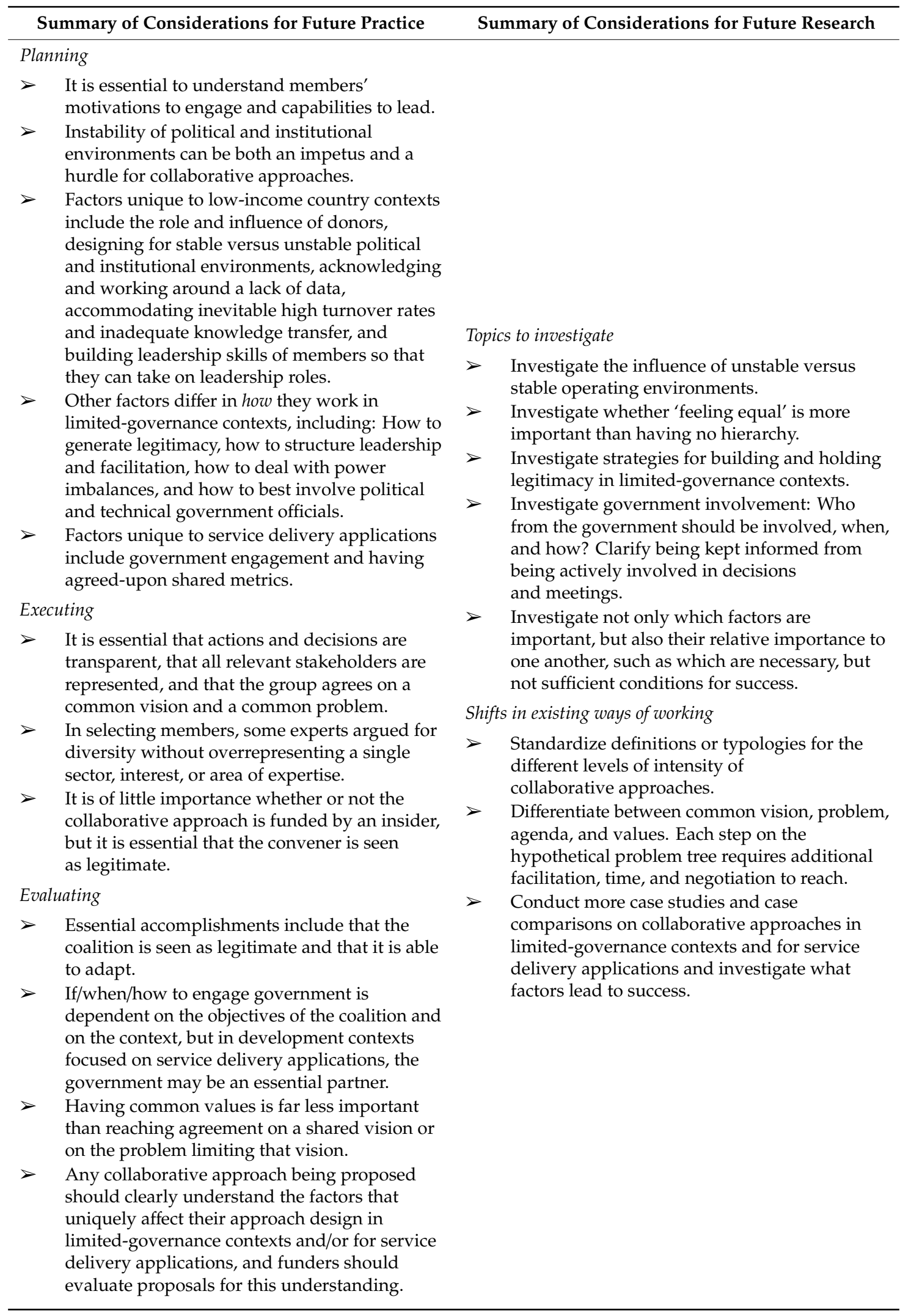




\section{References}

1. Kushner, D.C.; MacLean, L.M. Introduction to the Special Issue: The Politics of the Nonstate Provision of Public Goods in Africa. Afr. Today 2015, 62, 7-17. [CrossRef]

2. Katusiimeh, M.W. The Nonstate Provision of Health Services and Citizen Accountability in Uganda. Afr. Today 2015, 62, 85. [CrossRef]

3. Kyessi, A.G. Community-based urban water management in fringe neighbourhoods: The case of Dar es Salaam, Tanzania. Habitat Int. 2005, 29, 1-25. [CrossRef]

4. Darteh, B.; Moriarty, P.; Huston, A. How to Use Learning Alliances to Achieve Systems Change at Scale; IRC: The Hague, The Netherlands, 2019; p. 31.

5. USAID. Local Systems: A Framework for Supporting Sustained Development; USAID: Washington, DC, USA, 2014; p. 23.

6. USAID. Self Reliance Learning Agenda Fact Sheet: Evidence to Support the Journey to Self Reliance. 2019. Available online: https://www.usaid.gov/documents/1870/self-reliance-learning-agenda-fact-sheet (accessed on 16 September 2019).

7. Kaufmann, D.; Kraay, A. Worldwide Governance Indicators; World Bank: Washington, DC, USA, 2018.

8. Mo Ibrahim Foundation. African Governance Report: Agendas 2063 \& 2030: Is Africa on Track? Mo Ibrahim Foundation: London, UK, 2019.

9. Kaufmann, D.; Kraay, A.; Mastruzzi, M. The Worldwide Governance Indicators: Methodology and Analytical Issues. Hague J. Rule Law 2011, 3, 220-246. [CrossRef]

10. Kania, J.; Kramer, M.; Collective Impact. In Stanford Social Innovation Review; 2011; Winter, pp. 36-41. Available online: https://senate.humboldt.edu/sites/default/files/senate/Chair\%20Written\%20Report\%201-232018.pdf (accessed on 24 March 2020).

11. Tetra Tech. AguaConsult Beyond Collaboration: Learning from National and District-level Collective Action Efforts in WASH; IRC International Water and Sanitation Centre: The Hague, The Netherlands, 2019; pp. 58-59.

12. Kekez, A.; Howlett, M.; Ramesh, M. Collaboration in public service delivery: What, when and how. In Collaboration in Public Service Delivery; Edward Elgar Publishing: Cheltenham, UK, 2019; pp. 2-19, ISBN 978-1-78897-858-3.

13. Heath, R.G.; Isbell, M.G. Interorganizational Collaboration: Complexity, Ethics, and Communication; Waveland Press Inc.: Long Groce, IL, USA, 2017; ISBN 978-1-4786-3293-1.

14. Koschmann, M.A.; Sanders, M. Understanding Nonprofit Work: A Communication Perspective; Wiley-Blackwell: Hoboken, NJ, USA, 2020; ISBN 978-1-119-43125-1.

15. Gray, B.; Purdy, J. Collaborating for Our Future: Multistakeholder Partnerships for Solving Complex Problems; Oxford Scholarship Online: Oxford, UK, 2018; ISBN 978-0-19-878284-1.

16. Gray, B. Collaborating: Finding Common Ground for Multiparty Problems, 1st ed.; Jossey-Bass Management Series; Jossey-Bass: San Francisco, CA, USA, 1989; ISBN 978-1-55542-159-5.

17. Koschmann, M.A. The Communicative Accomplishment of Collaboration Failure: Collaboration Failure. J. Commun. 2016, 66, 409-432. [CrossRef]

18. Ansell, C.; Gash, A. Collaborative Governance in Theory and Practice. J. Public Adm. Res. Theory 2007, 18, 543-571. [CrossRef]

19. Emerson, K.; Nabatchi, T.; Balogh, S. An Integrative Framework for Collaborative Governance. J. Public Adm. Res. Theory 2012, 22, 1-29. [CrossRef]

20. Huxham, C. Theorizing collaboration practice. Public Manag. Rev. 2003, 5, 401-423. [CrossRef]

21. Lewis, L.K. Collaborative interaction: Review of communication scholarship and a research agenda. Ann. Int. Commun. Assoc. 2006, 30, 197-247. [CrossRef]

22. Owusu, F.; Ohemeng, F.L.K. The Public Sector and Development in Africa: The Case for a Developmental Public Service. In Rethinking Development Challenges for Public Policy: Insights from Contemporary Africa; Palgrave Macmillan: London, UK, 2012; pp. 117-154.

23. Larbi, G.A. Institutional constraints and capacity issues in decentralizing management in public services: The case of health in Ghana. J. Int. Dev. 1998, 10, 377-386. [CrossRef]

24. Post, A.E.; Bronsoler, V.; Salman, L. Hybrid Regimes for Local Public Goods Provision: A Framework for Analysis. Perspect. Polit. 2017, 15, 952-966. [CrossRef] 
25. Hofstede, G. Motivation, leadership, and organization: Do American theories apply abroad? Organ. Dyn. 1980, 9, 42-63. [CrossRef]

26. Ibarra, H. National Cultures and Work-Related Values: The Hofstede Study; Harvard Business School: Boston, MA, USA, 1996.

27. Ayee, J.R.A. Improving Effectiveness of Africa's Public Sector. In Rethinking Development Challenges for Public Policy: Insights from Contemporary Africa; Palgrave Macmillan: London, UK, 2012; pp. 83-116.

28. Mxakato-Diseko, N.J. The Changing Role and Image of the Public Service in Africa. Presented at the Workshop for Enhancing the Performance of the African Public Service Commissions and Other Appointing Commissions/Authorities, Kampala, Uganda, 7-11 April 2008; p. 4.

29. Estrin, S.; Pelletier, A. Privatization in Developing Countries: What Are the Lessons of Recent Experience? World Bank Res. Obs. 2018, 33, 65-102. [CrossRef]

30. UNICEF; WHO. Progress on Household Drinking Water, Sanitation, and Hygiene 2000-2017; Special Focus on Inequalities: New York, NY, USA, 2019.

31. RWSN. Myths of the Rural Water Supply Sector. RWSN Perspectives; RWSN: Gallen, Switzerland, $2010 ;$ p. 7. Available online: https://www.rural-water-supply.net/en/resources/details/226 (accessed on 9 March 2019).

32. Brinkerhoff, D.W.; Brinkerhoff, J.M. Public Sector Management Reform in Developing Countries: Perspectives Beyond NPM Orthodoxy: Public Sector Management Reform. Public Adm. Dev. 2015, 35, 222-237. [CrossRef]

33. Moriarty, P.; Smits, S.; Butterworth, J.; Franceys, R. Trends in Rural Water Supply: Towards a Service Delivery Approach. Water Altern. 2013, 6, 21.

34. Bayliss, K. Utility privatisation in Sub-Saharan Africa: A case study of water. J. Mod. Afr. Stud. 2003, 41, 507-531. [CrossRef]

35. Acey, C. Hybrid Governance and the Human Right to Water. Plan. J. 2016, 28. [CrossRef]

36. Kajenthira, A.; Sion, P. Collective Impact without Borders. Available online: https://ssir.org/articles/entry/ collective_impact_without_borders (accessed on 9 January 2019).

37. Christens, B.D.; Inzeo, P.T. Widening the view: Situating collective impact among frameworks for community-led change. Commun. Dev. 2015, 46, 420-435. [CrossRef]

38. Hanleybrown, F.; Kania, J.; Kramer, M. Channeling Change: Making Collective Impact Work. 2012. Available online: https://ssir.org/articles/entry/channeling_change_making_collective_impact_work (accessed on 10 April 2018).

39. Talesh, S. Public law and regulatory theory. In Handbook on Theories of Governance; Edward Elgar Publishing: Cheltenham, UK, 2016; pp. 102-110, ISBN 978-1-78254-849-2.

40. Gray, B. Assessing inter-organizational collaboration: Multiple conceptions and multiple methods. In Cooperative Strategy: Economic, Business, and Organizational Issues; Oxford University Press Inc.: New York, NY, USA, 2000; pp. 243-260, ISBN 0-19-829689-4.

41. Keyton, J.; Ford, D.J.; Smith, F.L. A Mesolevel Communicative Model of Collaboration. Commun. Theory 2008, 18, 376-406. [CrossRef]

42. Schoon, M.; Cox, M. Collaboration, Adaptation, and Scaling: Perspectives on Environmental Governance for Sustainability. Sustainability 2018, 10, 679. [CrossRef]

43. Hrelja, R.; Pettersson, F.; Westerdahl, S. The Qualities Needed for a Successful Collaboration: A Contribution to the Conceptual Understanding of Collaboration for Efficient Public Transport. Sustainability 2016, 8, 542. [CrossRef]

44. Feiock, R.C. The Institutional Collective Action Framework: Institutional Collective Action Framework. Policy Stud. J. 2013, 41, 397-425. [CrossRef]

45. Margerum, R. Beyond Consensus; MIT Press: Cambridge, MA, USA, 2011.

46. Lasker, R.D.; Weiss, E.S.; Miller, R. Partnership Synergy: A Practical Framework for Studying and Strengthening the Collaborative Advantage. Milbank Q. 2001, 79, 179-205. [CrossRef] [PubMed]

47. Wolff, T. A Practitioner's Guide to Successful Coalitions. Am. J. Commun. Psychol. 2001, 29, $173-191$. [CrossRef] [PubMed]

48. Jackson, M.; Stewart, R.; Fielding, K.; Cochrane, J.; Beal, C. Collaborating for Sustainable Water and Energy Management: Assessment and Categorisation of Indigenous Involvement in Remote Australian Communities. Sustainability 2019, 11, 427. [CrossRef] 
49. Ayala-Orozco, B.; Rosell, J.; Merçon, J.; Bueno, I.; Alatorre-Frenk, G.; Langle-Flores, A.; Lobato, A. Challenges and Strategies in Place-Based Multi-Stakeholder Collaboration for Sustainability: Learning from Experiences in the Global South. Sustainability 2018, 10, 3217. [CrossRef]

50. Warner, J. Multi-Stakeholder Platforms for Integrated Water Management; Routledge: New York, USA, 2016.

51. Reid, S.; Hayes, J.P.; Stibbe, D. Platforms for Partnership: Emerging Good Practice to Systematically Engage Business as a Partner in Development; The Partnering Initiative: Oxford, UK, 2014. Available online: https:// www.thepartneringinitiative.org/wp-content/uploads/2015/03/PLATFORMStealcoverallpages.pdf (accessed on 24 March 2020).

52. Verhallen, A.; Warner, J.; Santbergen, L. Towards Evaluating MSPs for Integrated Catchment Management. In Multi-Stakeholder Platforms for Integrated Water Management; Routledge: New York, NY, USA, 2007; pp. 259-271.

53. Torgersen, G.-E. Interaction: "Samhandling" under Risk-A Step Ahead of the Unforeseen. Cappelen Damm Akademisk/NOASP: Oslo, Norway, 2018; ISBN 978-82-02-53502-5.

54. Mayan, M.; Pauchulo, A.L.; Gillespie, D.; Misita, D.; Mejia, T. The promise of collective impact partnerships. Commun. Dev. J. 2019. [CrossRef]

55. Cabaj, M.; Weaver, L. Collective Impact 3.0. In Using Collective Impact to Bring Community Change; Series: The Community Development Research and Practice Series; Routledge: New York, NY, USA, 2018; Volume 9 , pp. 97-112, ISBN 978-1-315-54507-3.

56. Blatz, J. Filling the Gaps in Collective Impact. Stanf. Soc. Innov. Rev. 2019, 5. Available online: https://ssir.org/articles/entry/filling_the_gaps_in_collective_impact?utm_source=newsletter\&utm_ medium =email\&utm_content=Article\%3A\%20Filling\%20the\%20Gaps\%20in\%20Collective\%20Impact\& utm_campaign=CIF2019VCKeyFactorsInvite\# (accessed on 26 November 2019).

57. Flood, J.; Minkler, M.; Hennessey Lavery, S.; Estrada, J.; Falbe, J. The Collective Impact Model and Its Potential for Health Promotion: Overview and Case Study of a Healthy Retail Initiative in San Francisco. Health Educ. Behav. 2015, 42, 654-668. [CrossRef]

58. Walzer, N.; Weaver, L.; McGuire, C. Collective impact approaches and community development issues. Commun. Dev. 2016, 47, 156-166. [CrossRef]

59. The CEO Water Mandate. Guide to Water-Related Collective Action; UN Global Compact: New York, NY, USA, 2013; p. 56.

60. Dalkey, N.; Helmer, O. An Experimental Application of the DELPHI Method to the Use of Experts. Manag. Sci. 1963, 9, 458-467. [CrossRef]

61. Perveen, S.; Kamruzzaman, M.D.; Yigitcanlar, T. Developing Policy Scenarios for Sustainable Urban Growth Management: A Delphi Approach. Sustainability 2017, 9, 1787. [CrossRef]

62. Henning, J.; Jordaan, H. Determinants of Financial Sustainability for Farm Credit Applications-A Delphi Study. Sustainability 2016, 8, 77. [CrossRef]

63. Jordan, E.; Javernick-Will, A. Indicators of Community Recovery: Content Analysis and Delphi Approach. Nat. Hazards Rev. 2013, 14, 21-28. [CrossRef]

64. Hallowell, M.R.; Gambatese, J.A. Qualitative Research: Application of the Delphi Method to CEM Research. J. Constr. Eng. Manag. 2010, 136, 99-107. [CrossRef]

65. Gray, B.; Wood, D.J. Collaborative alliances: Moving from practice to theory. J. Appl. Behav. Sci. 1991, 27, 3-22. [CrossRef]

66. Ansell, C.; Gash, A. Collaborative Platforms as a Governance Strategy. J. Public Adm. Res. Theory 2018, 28, 16-32. [CrossRef]

67. Emerson, K.; Nabatchi, T. Collaborative Governance Regimes; Public Management and Change Series; Georgetown University Press: Washington, DC, USA, 2015; ISBN 978-1-62616-252-5.

68. Dedoose. 2.14 Web Application for Managing Analyzing and Presenting Qualitative and Mixed Method Research Data; Version 8; SocioCultural Research Consultants, LLC: Los Angeles, CA, USA, 2019.

69. Blackstone, A. Chapter 11: Unobtrusive Research. In Principles of Sociological Inquiry-Qualitative and Quantitative Methods; Saylor Foundation: Washington, DC, USA, 2018; ISBN 978-1-4533-2889-7.

70. Nkum Associates. Research on Learning Alliance Approach; Triple S Project Report; IRC Ghana: Accra, Ghana, 2014.

71. Smits, S.; Moriarty, P. Learning Alliances: Scaling Up Innovations in Water, Sanitation and Hygiene; IRC International Water and Sanitation Centre: Delft, The Netherlands, 2007; ISBN 978-90-6687-056-7. 
72. Schmidt, R.C. Managing Delphi Surveys Using Nonparametric Statistical Techniques. Decis. Sci. 1997, 28, 763-774. [CrossRef]

73. Von der Gracht, H.A. Consensus measurement in Delphi studies. Technol. Forecast. Soc. Chang. 2012, 79, 1525-1536. [CrossRef]

74. Naylor, C.D.; Basinski, A.; Baigrie, R.S.; Goldman, B.S.; Lomas, J. Placing patients in the queue for coronary revascularization: Evidence for practice variations from an expert panel process. Am. J. Public Health 1990, 80, 1246-1252. [CrossRef]

75. Cafiso, S.; Di Graziano, A.; Pappalardo, G. Using the Delphi method to evaluate opinions of public transport managers on bus safety. Saf. Sci. 2013, 57, 254-263. [CrossRef]

76. Gillam, R.J.; Counts, J.M.; Garstka, T.A. Collective impact facilitators: How contextual and procedural factors influence collaboration. Commun. Dev. 2016, 47, 209-224. [CrossRef]

77. Ulibarri, N. Tracing Process to Performance of Collaborative Governance: A Comparative Case Study of Federal Hydropower Licensing: Ulibarri: A Comparative Case Study of Federal Hydropower Licensing. Policy Stud. J. 2015, 43, 283-308. [CrossRef]

78. Ofoegbu, C.; New, M.; Staline, K. The Effect of Inter-Organisational Collaboration Networks on Climate Knowledge Flows and Communication to Pastoralists in Kenya. Sustainability 2018, 10, 4180. [CrossRef]

(C) 2020 by the authors. Licensee MDPI, Basel, Switzerland. This article is an open access article distributed under the terms and conditions of the Creative Commons Attribution (CC BY) license (http://creativecommons.org/licenses/by/4.0/). 